\title{
TOPOLOGICAL LOCALIZATION AND NILPOTENT GROUPS
}

\author{
BY PETER HILTON ${ }^{1}$, GUIDO MISLIN ${ }^{2}$ AND JOSEPH ROITBERG ${ }^{3}$
}

Communicated by Morton Curtis, May 5, 1972

I. The theory of topological localization has been developed in various forms by several authors (cf. for example [1], [2], [8], [10], [12]). In this note, we place ourselves in the framework of $[10]$ and announce some foundational results in the theory. The importance and usefulness of these results has already been well illustrated, for example, by the applications in [4], [5], [9].

Before stating our main result, we fix some notation. Let $X$ be a connected, simple CW-complex of finite type. If $P$ is a collection of primes, we denote by $X_{P}$ the localization of $X$ at $P$, by $e_{P}: X \rightarrow X_{P}$ the localization map, by $X_{0}$ the rationalization of $X$, and by $r_{P}: X_{P} \rightarrow X_{0}$ the rationalization map, characterized by the equation $r_{P} \circ e_{P}=e_{0} \quad[10]$. We then have

THEOREM 1. Let $W$ be a connected, finite $C W$-complex and $X$ a connected, simple $C W$-complex of finite type. Then the natural map

$$
\Phi:[W, X] \rightarrow \prod_{p}\left(\left[W, X_{p}\right] \stackrel{r_{p}^{\prime}}{\longrightarrow}\left[W, X_{0}\right]\right)
$$

from the (pointed) homotopy set $[W, X]$ into the pullback, over all primes $p$, of the maps $r_{p}^{\prime}$, induced by $r_{p}$, is a set-theoretic bijection.

The surjectivity of $\Phi$ is an easy consequence of Theorem 2 below and known results about localization [10]. The injectivity of $\Phi$ may be established by elementary arguments in abelian group theory provided $W$ is a sphere, or more generally a double suspension. A more delicate argument, but still requiring only abelian group theory, was introduced by Mimura-Nishida-Toda [8] to prove the injectivity of $\Phi$ for $W$ a suspension. We show that the general case, with no added restriction on $W$, leads naturally to a problem in the theory of nilpotent groups. The connection between our problem and the theory of nilpotent groups stems from a classical result of G. W. Whitehead [11] asserting that, for $W$ a connected finite complex and $G$ a topological group of finite type, the (pointed) homotopy set $[W, G]$ admits naturally the structure of a finitely generated nilpotent group. The solution of our problem is strongly

AMS 1970 subject classifications. Primary 55D99.

1 The first author was partially supported by NSF grant GP-29107.

2 The second author was partially supported by NSF grant GP-29544X.

3 The third author was partially supported by NSF grant GP-29431. 
suggested by the algebraic theory of $P$-localization of nilpotent groups, due to Malcev and Lazard [7]. The bridge between this algebraic theory and homotopy theory is supplied by Jerome Harrison's observation ${ }^{4}[3]$ that, if $G$ is connected, the nilpotent group $\left[W, G_{P}\right]$ is naturally isomorphic to $[W, G]_{P}$, the $P$-localization of the nilpotent group $[W, G]$, where $W$ and $G$ are as above.

In $\$ 2$ we indicate briefly how to extend the approach of [8] to show that $\operatorname{ker} \Phi=0$. If $W$ is a suspension (the case considered in [8]), this is of course equivalent to the injectivity of $\Phi$; but in general it is a priori a weaker result, owing to the absence of a group structure on $[W, X]$. This weaker result nevertheless suffices for the applications envisaged in [4], [5].

A detailed proof of Theorem 1, based on rather different techniques from those sketched below (but also involving nilpotent group theory), will appear in [6]. Further applications, particularly to the theory of $H$-spaces, which demand the full strength of Theorem 1 , will also be discussed in [6].

II. The following "stability" or "finiteness" theorem is useful for our purposes and seems also to possess some intrinsic interest. A proof will be found in [6].

THEOREM 2. Let $W$ be a connected, finite $C W$-complex, $X$ a connected, simple $C W$-complex of finite type and let $f: W \rightarrow X_{0}$. If $P_{k}$ denotes the collection of all but the first $k$ primes, then there exists an integer $l>0$ for which there exists a unique $g: W \rightarrow X_{P_{l}}$ satisfying $r_{P_{l}} \circ g=f$.

We now sketch briefly a proof that $\operatorname{ker} \Phi=0$, following the lines of [8]. Thus we have a map $\operatorname{map}^{5} f \in[W, X]$ with $e_{p} \circ f=0$ for all $p$ and seek to prove $f=0$. Theorem 2 allows us to conclude the existence of an integer $l>0$ so that $e_{P_{l}} \circ f=0$. Hence we have a partitioning of the set $\Pi$ of all primes into finitely many disjoint subsets, namely ${ }^{6}$ $\left\{p_{1}\right\}, \ldots,\left\{p_{l}\right\}, P_{l}$, such that, for each of these subsets $P$, we have $e_{P} \circ f=0$. We may therefore proceed by showing that if $P$ and $Q$ are disjoint sets of primes, then $e_{P \cup Q} \circ f=0$ provided $e_{P} \circ f=0, \quad e_{Q} \circ f=0$. For, by the above, and the fact that $X_{\Pi}=X, e_{\Pi}=1$, we are in a position to invoke finite induction to deduce that $f=0$.

By carefully examining the proof of Theorem 4.7 of [8], it is not difficult to see that we are thus led to the following situation.

Let $s_{P}^{\prime}:\left[W, \Omega X_{P}\right] \rightarrow\left[W, \Omega X_{0}\right]$ be the map induced by $s_{P}=\Omega r_{P}$ :

${ }^{4}$ We are indebted to Hans Scheerer for providing us with copies of this work.

${ }^{5}$ Here, and elsewhere, we deliberately confuse maps with their homotopy classes.

6 We denote the $i$ th prime by $p_{i}$. 
$\Omega X_{P} \rightarrow \Omega X_{0}$; similarly for $s_{Q}^{\prime}$. We must then show that, given $h \in\left[W, \Omega X_{0}\right]$, there exist $j \in\left[W, \Omega X_{P}\right], \quad k \in\left[W, \Omega X_{Q}\right]$ such that

$$
h=s_{P}^{\prime}(j) \cdot s_{Q}^{\prime}(k) \text {. }
$$

Now the essential point to note in this connection is that, for any $h \in\left[W, \Omega X_{0}\right]$, some integral power $h^{n}$ is induced from $[W, \Omega X]$, i.e. $h^{n}=u_{0}$, where $u_{0}$ is induced from an element $u$ in $[W, \Omega X]$ by means of the rationalization $e_{0}: X \rightarrow X_{0}$. It is possible to prove this directly, but essentially the same reasoning shows that $\left[W, \Omega X_{0}\right]$ is in fact the Malcev localization $^{7}$ of $[W, \Omega X] \quad[3]$. Thus we have

$$
h=u_{0}^{1 / n}, \quad u \in[W, \Omega X],
$$

and by a standard argument, we may write

$$
u_{0}^{1 / n}=u_{0}^{\rho} \cdot u_{0}^{\sigma}, \quad \rho \in \mathbf{Z}_{P}, \quad \sigma \in \mathbf{Z}_{Q} .
$$

As $\left[W, \Omega X_{P}\right]$ is a $P$-local nilpotent group (in fact, the Lazard $P$-localization of $[W, \Omega X][3])$, it follows that $u_{0}^{\rho} \in \operatorname{im} s_{P}^{\prime}$. Similarly, $u_{0}^{\sigma} \in$ im $s_{Q}^{\prime}$, and the argument is completed.

As a final comment, we remark that there is an analogue of Theorem 1 with $p$-profinite completion replacing $p$-localization. A significant point to note here is that the group $\left[W, G_{P}\right]$, where $G_{P}$ is the $P$-profinite completion of the space $G([10],[2])$, is naturally isomorphic to $[W, G]_{P}$, the $P$-profinite completion of the group $[W, G]$. This fact may be deduced along the lines of Harrison's argument in the local situation, making use of the fact that profinite completion is an exact functor on the category of finitely generated nilpotent groups.

\section{BIBLIOGRAPHY}

1. A. K. Bousfield and D. M. Kan, Homotopy with respect to a ring, Proc. Sympos. Pure Math., vol. 22, Amer. Math. Soc., Providence, R.I., 1971, pp. 59-64.

2. Localization and completion in homotopy theory, Bull. Amer. Math. Soc. 77 (1971), 1006-1010.

3. J. Harrison and $\mathbf{H}$. Scheerer, Mimeographed notes.

4. P. J. Hilton, G. Mislin and J. Roitberg, Sphere bundles over spheres and non-cancellation phenomena, J. London Math. Soc. (to appear); See also, Lecture Notes in Math., vol. 249, Springer-Verlag, Berlin and New York, 1971, pp. 34-46.

5. - H-spaces of rank 2 and non-cancellation phenomena, Invent. Math. (to appear).

6. - Homotopical localization, J. London Math. Soc. (to appear).

7. M. Lazard, Sur les groupes nilpotents et les anneaux de Lie, Ann. Sci. École Norm. Sup. (3) 71 (1954), 101-190. MR 19, 529.

8. M. Mimura, G. Nishida and $\mathrm{H}$. Toda, Localization of $\mathrm{CW}$-complexes and its applications, J. Math. Soc. Japan 23 (1971), 593-624.

9. G. Mislin, The genus of an H-space, Lecture Notes in Math., vol. 249, Springer-Verlag, Berlin and New York, 1971, pp. 75-83.

\footnotetext{
${ }^{7}$ Malcev calls this 'completion', but we prefer the term 'localization' or 'rationalization' to avoid confusion with profinite completion.
} 
10. D. Sullivan, Geometric topology. I: Localization, periodicity and Galois symmetry, Mimeographed notes, M.I.T., Cambridge, Mass., 1970.

11. G. W. Whitehead, On mappings into group-like spaces, Comment Math. Helv. 28 (1954), 320-328. MR 16, 505.

12. A. Zabrodsky, Homotopy associativity and finite $C W$-complexes, Topology 9 (1970), 121-128. MR 42 \#1118.

Battelle Memorial Institute, 4000 Ne 41st Street, Seattle, Washington 98105 and Department of Mathematics, University of Washington, Seattle, Washington 98195 (Current address of Peter Hilton)

Department of Mathematics, Eth, Zurich, Switzerland (Current address of Guido Mislin)

Department of Mathematics, State University of New York at Stony Brook, STONY BROOK, NEW YORK 11790

Current address (Joseph Roitberg): Department of Mathematics, Hunter College, College University of New York, New York, New York 10021 